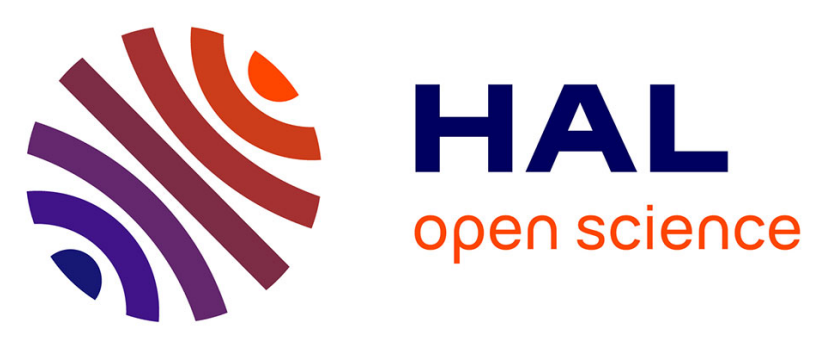

\title{
Single particle-tracking method in fluorescence microscopy to monitor bioenergetic responses of individual mitochondria
}

Camille Colin, Emmanuel Suraniti, Emma Abell, Audrey Sémont, Neso Sojic, Philippe Diolez, Stéphane Arbault

\section{To cite this version:}

Camille Colin, Emmanuel Suraniti, Emma Abell, Audrey Sémont, Neso Sojic, et al.. Single particletracking method in fluorescence microscopy to monitor bioenergetic responses of individual mitochondria. Mitochondrial Medicine, 2276, Springer US, pp.153-163, 2021, Methods in Molecular Biology, 10.1007/978-1-0716-1266-8_11. hal-03424380

\author{
HAL Id: hal-03424380 \\ https://hal.science/hal-03424380
}

Submitted on 15 Dec 2021

HAL is a multi-disciplinary open access archive for the deposit and dissemination of scientific research documents, whether they are published or not. The documents may come from teaching and research institutions in France or abroad, or from public or private research centers.
L'archive ouverte pluridisciplinaire HAL, est destinée au dépôt et à la diffusion de documents scientifiques de niveau recherche, publiés ou non, émanant des établissements d'enseignement et de recherche français ou étrangers, des laboratoires publics ou privés. 


\title{
Single particle-tracking method in fluorescence microscopy to monitor bioenergetic responses of individual mitochondria
}

\author{
Camille COLIN, ${ }^{1,2}$ Emmanuel SURANITI, ${ }^{1}$ Emma ABELL, ${ }^{2}$ Audrey SÉMONT ${ }^{2}$, \\ Neso SOJIC, ${ }^{1}$ Philippe DIOLEZ, ${ }^{2}$ Stéphane ARBAULTT ${ }^{1, *}$
}

Address:

1. Univ. Bordeaux, CNRS, INP Bordeaux, ISM, UMR 5255, NSysA group, 33405 Talence, FRANCE

2. INSERM U1045 - Centre de Recherche Cardio-Thoracique de Bordeaux \& LIRYC - Institut de Rythmologie et Modélisation Cardiaque, Université de Bordeaux, France, $\mathrm{CHU}$ de Bordeaux, France

\section{Summary}

The spectroscopic methods commonly used to study mitochondria bioenergetics do not show the diversity of responses within a population of mitochondria (isolated or in a cell), and/or cannot measure individual dynamics. New methodological developments are necessary in order to improve quantitative and kinetic resolutions and eventually gain further insights on individual mitochondrial responses, such as studying activities of the mitochondrial Permeability Transition Pore (mPTP). The work reported herein is devoted to study responses of single mitochondria within a large population after isolation from cardiomyocytes. Mitochondria were pre-loaded with a commonly used membrane potential-sensitive dye (TMRM), they are then deposited on a plasma-treated glass coverslip and subsequently energized or inhibited by additions of usual bioenergetics effectors. Responses were analysed by fluorescence microscopy over few thousands mitochondria simultaneously with a single organelle resolution. We 
report an automatic method to analyse each image of time-lapse stacks based on the Trackmate-ImageJ plugin and specially made Python scripts. Images are processed to eliminate defects of illumination inhomogeneity, improving by at least two orders of magnitude the Signal/Noise ratio. This method enables us to follow the track of each mitochondrion within the observed field and monitor its fluorescence changes, with a time resolution of $400 \mathrm{msec}$, uninterrupted over the course of the experiment. Such methodological improvement is a pre-requisite to further study the role of MPTP in single mitochondria during calcium transient loading.

Keywords: Mitochondria; Fluorescence microscopy; Membrane potential; Bioenergetics; Single organelle; Single particle tracking; Fiji software; TrackMate.

Running head: Single particle tracking

\section{Introduction}

The monitoring by microscopy of mitochondria, when isolated or when forming a network in cells, constitutes a major approach to resolve their organization, functioning and activity under physiological (1) or pathological $(2,3)$ situations. Owing to the recent developments of microscopy techniques, these are increasingly used since they allow to follow-up responses in living cells or monitor resolved kinetics on minute sample quantities under a non-invasive manner. NADH and FAD auto-fluorescence can be used to monitor endogenously the mitochondrial bioenergetics when modulations of the Respiratory Chain are used. A large variety of fluorescent dyes can also be found to assess mitochondrial functions (membrane potential, ROS, $\mathrm{pH} . .$.$) , their number continuously increasing in literature (4). The most representative$ family is the one dedicated to monitor $\Delta \Psi$ with high sensitivity and kinetic resolution, which includes dyes quoted as JC1, TMRE or TMRM.(5-8)

Microscopy approaches are used first for imaging and to a lower extent to resolve metabolic responses such as depolarization waves or transients by mitochondria under bioenergetic variations. There are now multiple reports at the level of single mitochondrion resolution from experiments in cells, notably in cardiomyocytes thanks to their organization, $(9,10)$ or for isolated mitochondria deposited on various supports (glass coverslip, modified or not, PDMS, etc.).(11-13) However, only few remarkable events at single mitochondria are usually reported and shown in communications, while the response of populations, networks with an individual resolution would help in deciphering mechanisms and 
discriminating the existence of sub-populations (heteroplasmy, metabolic variations) (14). To do so, automated image processing and data analysis protocols still need to be developed to promote large scale studies and statistics from individual mitochondria responses in microscopy; this is the aim of the present methodological development. We studied responses to bioenergetics effectors of mitochondria isolated from rat cardiomyocytes. After depositing on a glass coverslip, their membrane potential evolutions were monitored simultaneously from thousands of entities with a single mitochondrion resolution. This was made possible with microscopy image corrections and improvements (Fiji software functions) along with individual follow-up of objects over time and distance (TrackMate plug-in) and additional personalized scripts to generate matrices of data. All developed protocols are available free of charge from the authors upon request.

\section{Materials}

\subsection{Solutions}

1. Extraction buffer: Saccharose $300 \mathrm{mM}$, Tris- $\mathrm{HCl} 10 \mathrm{mM}, \mathrm{EGTA} 50 \mathrm{mM}, \mathrm{pH}$ adjusted to7.2 with $\mathrm{NaOH} 0.5 \mathrm{M}$.

2. Digestion buffer: Saccharose $300 \mathrm{mM}$, Tris- $\mathrm{HCl} 10 \mathrm{mM}$, EGTA $50 \mathrm{mM}$, Protease $0.1 \mathrm{mg} \cdot \mathrm{ml}^{-1}$ (from Streptomyces griseus, Type XIV, ref: P5147), pH 7.2.

3. Homogenization buffer: Saccharose 300 mM, Tris- HCl 10 mM, EGTA 50 mM, BSA 0.2 \%, pH adjusted to 7.2 with $\mathrm{NaOH} 0.5 \mathrm{M}$.

4. Respiration buffer: Saccharose $300 \mathrm{mM}, \mathrm{KCl} 100 \mathrm{mM}$, EGTA $1 \mathrm{mM}, \mathrm{MgCl} 220 \mathrm{mM}, \mathrm{KH}_{2} \mathrm{PO}_{4} 10$ $\mathrm{mM}, \mathrm{BSA} 0.1 \%, \mathrm{pH}$ adjusted to 7.2 with $\mathrm{NaOH} 0.5 \mathrm{M}$.

5. Glutamate, malate, succinate (ref: G1626, M9138, S2378, respectively) stock solutions are prepared at $500 \mathrm{mM}$ in Respiration buffer.

6. ADP (Adenosine diphosphate, ref A2754) stock solution is prepared at $100 \mathrm{mM}$ in Respiration buffer.

7. Rotenone (ref R8875) stock solution is prepared at $1 \mathrm{mM}$ in DMSO (see Note 1).

8. Oligomycin (ref 75351) stock solution is prepared at $1 \mathrm{mM}$ in methanol (see Note 1).

9. C-ATR (carboxy-atractyloside potassium salt, ref C4992) stock solution is prepared at $5 \mathrm{mM}$ in Respiration buffer (see Note 1). 
10. CCCP (Carbonyl cyanide 3-chlorophenylhydrazone, ref C2759) stock solution is prepared at $1 \mathrm{mM}$ in acetone (see Note 1).

\subsection{Fluorescence Imaging}

1. Experiments were performed with an inverted epifluorescence microscope from Leica@ (DMI 6000 B model) equipped with a 40x objective (dry; NA: 0.75; Leica HC PL APO), and a camera from Hamamatsu@ (ORCA-Flash4.0).

2. Images were collected via MetaMorph (Molecular Devices (C) and analyzed with Fiji - ImageJ (NIH free supply) softwares.

\section{Methods}

\subsection{Rat heart extraction}

1. Mitochondria are extracted from the heart of Wistar male rats (from Janvier labs, France) according to the following procedure (see Note 2).

2. The rat is anaesthetised by inhalation of isoflurane (4\%) in an induction chamber for 2 minutes.

3. Once anaesthesia observed, the rat is weighted and injected subcutaneously with $0.1 \mathrm{~mL}$ of Heparin at 5000 U. $\mathrm{mL}^{-1}$.

4. The rat is placed back in the induction chamber ( $4 \%$ isoflurane) for a further 3 minutes and euthanized by cervical dislocation.

5. The heart is rapidly extracted from the thorax and placed in cold Extraction buffer, to stop contractions (see Note 2).

6. The heart is rinsed with Extraction buffer, weighed, and unwanted tissue is removed.

\subsection{Extraction of cardiac mitochondria}

1. Ventricular tissue is excised and thoroughly minced with sharp scissors.

2. Minced tissue is transferred to a beaker containing $30 \mathrm{~mL}$ of Digestion buffer and placed on a magnetic stirrer for 7 minutes. 
3. The solution is transferred to a glass-Teflon homogeniser, remaining Digestion buffer is used to remove any tissue left in beaker. The partially digested tissue is homogenized for 3 minutes, to break up any remaining tissu.

4. The homogenate is centrifuged at $7500 \mathrm{~g}$ for 7 minutes.

5. he supernatant is discarded, and the pellet re-suspended in $30 \mathrm{~mL}$ of Homogenization buffer, homogenised for 2 minutes and transferred to a new centrifuge tube.

6. The solution is centrifuged at $680 \mathrm{~g}$ for 10 minutes. The supernatant is then filtered with a nylon filter and transferred into a new centrifuge tube.

7. The solution is centrifuged at $7000 \mathrm{~g}$ for 10 minutes. The supernatant is discarded, and the pellet re-suspended in $40 \mu \mathrm{L}$ Homogenization buffer.

8. Mitochondrial protein quantification is performed with a classic Bradford assay, the resulting optical density is measured at $595 \mathrm{~nm}$ with a spectrophotometer (see Note 3).

\section{3. coverslip preparation}

1. Coverslips (12 mm diameter) are purchased from Fischer Scientific (Carolina Science \& Math manufacturer; ref. NC9537307).

2. Coverslips are treated just before use with a low pressure-oxygen plasma generator from Harrick Plasma@ (ref. plasma cleaner) at 300 mTorr, $100 \% \mathrm{O}_{2}$, during 10 minutes (see Note 4).

\subsection{Experimental Procedure for mitochondria imaging}

1. Before the experiment, a diluted solution of mitochondria is prepared at $0.1 \mathrm{mg} \cdot \mathrm{mL}^{-1}$ in Respiration buffer (see Note 5), supplemented with $10 \mathrm{nM}$ of TMRM (Tetramethylrhodamine methyl ester perchlorate, purchased from Sigma, ref T5428).

2. After 10 minutes of incubation, $600 \mu \mathrm{L}$ of the solution is deposited on the coverslip previously mounted on the microscope stage (see Note 6).

3. In bright field mode, the focus is on the top surface of the coverslip, and a 20 minutes-interval is allowed for mitochondria to sediment (see Note 7).

\subsection{Imaging of mitochondria}

1. The typical sequence for mitochondria imaging is the following: images are captured every 10 seconds during 20 minutes; exposure times are 40 milliseconds to detect either TMRM 
fluorescence in mitochondria or for additional observations in bright field. Fluorescence images are obtained with a fibered light source (Leica, ref EL6000) set at its minimum power, while white light images were obtained with a fibered LED source (CoolLed, ref PE100) connected to the microscope.

2. Observations are achieved using a 40x objective (dry; N.A. 0.75 ), allowing large field views (332.8*322.8 $\mu \mathrm{m}$ area) and single mitochondria measurements.

3. N2.1-type filter (Leica) is used for TMRM detection (excitation: $515-560 \mathrm{~nm}$, emission, long- pass $>590 \mathrm{~nm}$ ), and A-type filter (Leica) is used for endogenous NADH detection (excitation: 340-380 $\mathrm{nm}$, emission: long-pass $>425 \mathrm{~nm}$ ).

4. The following sequence of solutions is used to induce the different bioenergetic-stationary states in mitochondria: 1) addition of respiratory substrates; either glutamate plus malate at 5 $\mathrm{mM}$ each, or succinate at $5 \mathrm{mM}$ plus rotenone at $2 \mu \mathrm{M}$, or a combination of glutamate, malate and succinate, all at $5 \mathrm{mM} ; 2$ ) addition of ADP at $1 \mathrm{mM} ; 3$ ) addition of oligomycin at $5 \mu \mathrm{M}$ or CATR at $5 \mu \mathrm{M} ; 4)$ addition of CCCP at $0.5 \mu \mathrm{M}$.

5. 10 images are taken for each step of the sequence (100 s), note that 10 images are taken before adding the first substrate.

\subsection{Treatment of images with "Fiji"}

1. Before any data analysis, all images are stacked (image/stack/images to stack) in a file (Stack 1; Figure 1a) and duplicated (Stack 2; image/duplicate). A profile of the intensity variation versus a linear ROI (region of interest) can be drawn to check for the Signal/Noise ratio of the object detection (Figure 1b).

2. On Stack 2, a Gaussian-type filter is applied with a factor of 60 to correct a shading effect (process/filters/Gaussian Blur) due to the illumination inhomogeneity of the lamp on the epifluorescence microscope.

3. The obtained Stack 2 (Figure 1c) is subtracted from the original Stack 1 (image/process/image calculator/subtract) to generate a corrected Stack 3 (Figure 1d).

4. A median filter with a factor of 2 is applied to Stack 3 to eliminate noise resulting from the subtraction (image/process/filters/median) and enhance the signal-noise ratio. This produces a final Stack 4 (Figure 1e) used for further analyses. The result of the procedure on the image of a single mitochondrion is shown in Figure $\mathbf{1} \mathbf{f}$ to compare with the original one in Figure $\mathbf{1 b}$.

\subsection{Mitochondria follow-up with "TrackMate"}


1. On Stack 4, a Fiji software plug-in called "TrackMate" (15) is applied (plugin/Tracking/TrackMate). This plug-in enables to track the path of each individual mitochondrion over distance and time, according to two main steps.

2. The first step is to detect on each image all possible objects that can be classified as mitochondria. To do so, the "DoG detector" method is used with an "Estimated blob diameter" of 10 pixels, equivalent to $1.625 \mu \mathrm{m}$, and an intensity threshold between 1 to $5 \mathrm{AU}$. This method uses a difference of Gaussian fits to detect particles, and is optimal for spots of small size (see Note 8).

3. The second step is aimed at identifying the same object over time; for this purpose, the "simple LAP Tracker" method is used (see Note 9). According to their identification, provided as a number assigned by TrackMate (see Note 10), a follow-up of the displacement of individual mitochondria is possible (Figure 2a) though some boundaries need to be given: "Linking max distance" of 15 pixels, or $2.4375 \mu \mathrm{m}$; a "Gap-closing max distance" of 15 pixels or $2.4375 \mu \mathrm{m}$ and a "Gap-closing max frame gap" of 50 frames. Two different types of displacements of mitochondria on the surface along an experiment are compared in Figure $\mathbf{2 b}$.

4. Finally, the function "Analysis" is used to generate "Spots in track statistics" datasheets allowing further data analyses of displacements, intensity variations, etc.

\subsection{Python scripts for quantitative analyses}

1. Specialized informatics scripts were written with "Python" 4.0, "Spyder" environment and "Pandas, Numpy and SciPy" libraries, to automatically process the analysis of data. These scripts are available free of charge upon request from the authors.

2. At the beginning of each script, two filters are used: 1) A filter of movement to discard mobile mitochondria (> $3.25 \mu \mathrm{m}$ displacement), which can distort the analysis and overall the statistics (example in Figure 2c); 2) A time filter, to eliminate mitochondria that are not present enough ( $<10$ images, 100 seconds) along the whole sequence.

3. The first script was designed to calculate for every image the mean intensity of all individual mitochondria (TMRM fluorescence) in order to apprehend the global variations of the sample (Figure 3a).

4. To do so, "Spots in tracks statistics" is read with the function "read_csv" and data are grouped for each image with the function ".groupby ('FRAME')". After, the mean intensity is calculated 
("numpy.mean") for each image. Other parameters can be implemented as the number of mitochondria detected ("len") and the standard deviation of means ("numpy.std").

5. A second script was designed to create a graphical representation of the fluorescence intensity variation over time for each identified mitochondrion ("TRACK_ID") (Figure 3b).

6. To do so, "Spots in tracks statistics" are read and the data for all images are grouped for each unique identification number of all detected objects with the function "groupby ('TRACK_ID')". Then, the fluorescence intensity evolution over time for each mitochondrion is displayed graphically and saved as a '.png' file with the identification number as title.

\section{Notes}

1. This is a hazardous material; So, protective clothing must be worn during preparation and manipulation.

2. It is necessary to maintain cold on ice all solutions and instruments before starting and during the procedure of rat heart extraction.

3. For the Bradford assay, there is a 10 minute-incubation period before reading the optical density at $595 \mathrm{~nm}$.

4. The treatment of the glass surface by an oxygen plasma $\left(100 \% \mathrm{O}_{2}\right)$ clearly improves its hydrophilicity. It is observed that the plasma encourages mitochondria to be immobile on the surface for longer times (at least $50 \mathrm{~min}$ ), which is a mandatory condition for the quantitative monitoring of their fluorescence during different steps, particularly when solutions (activators or inhibitors) are injected into the surrounding area.

5. Please note that the concentration of mitochondria is an important parameter: we observe for concentrations higher than $0.2 \mathrm{mg}^{\mathrm{mL}} \mathrm{L}^{-1}$ that the number of mitochondria on the coverslip is too high and hinders single particle tracking. On the contrary, for a concentration below 0.05 $\mathrm{mg} \cdot \mathrm{mL}^{-1}$ mitochondria are not viable, a sudden loss of TMRM fluorescence is often observed.

6. The coverslip has to be immobile throughout an experiment because of the 3D positionprecision requested for particle tracking; vacuum grease can be used to help sticking to the support on the microscope platform.

7. 20x objective can also be used to help finding the right focus, but is not suitable to monitor the fluorescence changes. On the other hand 63x or higher magnification objectives can be used to 
improve resolution but the number of mitochondria simultaneously observed may be too low to draw significant conclusions.

8. "TrackMate" can also give an estimation of the object diameter inside the region of interest. To be accurate, it is important that the "Estimated blob diameter" parameter value is superior to the size of mitochondria. Two distinct sizes of mitochondria were detected in our sample, around $0.8 \mu \mathrm{m}$ and $1.4 \mu \mathrm{m}$, which may correspond to the two functional populations described for cardiomyocytes (16).

9. The "simple LAP Tracker" method used is designed for particles with no fusion or fission behaviour, such as in our experimental case. "TrackMate" offers other methods to track particles depending on the behaviour; manual tracking is also possible.

10. "TrackMate" plug-in assigns a unique identification number to every mitochondrion, which facilitates the manipulation and analyses of results for large sets of data. This number is also used by our specialised scripts.

\section{Acknowledgement}

The project was financially supported by the ANR ("Agence Nationale pour la Recherche", project MITOCARD $n^{\circ}$ ANR-17-CE11-0041), the University of Bordeaux, the CNRS ("Centre National de la Recherche Scientifique") and the INSERM ("Institut National de la Santé Et de la Recherche Médicale"). CC acknowledges the University of Bordeaux (Interdisciplinary-pluridisciplinary PhD funding program 2016) for his PhD fellowship. AS acknowledges IHU-LIRYC (Pessac, France) for her PhD fellowship.

\section{References}

(1) De Giorgi F, Lartigue L and Ichas F (2000) Electrical coupling and plasticity of the mitochondrial network Cell Calcium 28 365-370

(2) Creed S and McKenzie M (2019) Measurement of Mitochondrial Membrane Potential with the Fluorescent Dye Tetramethylrhodamine Methyl Ester (TMRM): Methods and Protocols Methods in molecular biology (Clifton, N.J.) 1928: 69-76

(3) Quintanilla R A, Matthews-Roberson T A, Dolan P J and Johnson G V W (2009) Caspase-cleaved Tau Expression Induces Mitochondrial Dysfunction in Immortalized Cortical Neurons: implications for the pathogenesis of Alzheimer disease J. Biol. Chem. 284: 18754-18766 
(4) Jiang N, Fan J, Xu F, Peng X, Mu H, Wang J and Xiong X (2015) Ratiometric Fluorescence Imaging of Cellular Polarity: Decrease in Mitochondrial Polarity in Cancer Cells Angew. Chem. Int. Ed. 54: 25102514

(5) Perry S W, Norman J P, Barbieri J, Brown E B and Gelbard H A( 2011 )Mitochondrial membrane potential probes and the proton gradient: a practical usage guide BioTechniques 50: 98-115

(6) Scaduto R C and Grotyohann L W (1999) Measurement of Mitochondrial Membrane Potential Using Fluorescent Rhodamine Derivatives Biophys. J. 76: 469-477

(7) Figueira T R, Melo D R, Vercesi A E and Castilho R F (2012) Safranine as a Fluorescent Probe for the Evaluation of Mitochondrial Membrane Potential in Isolated Organelles and Permeabilized Cells Mitochondrial Bioenergetics Methods in Molecular Biology vol 810, ed C M Palmeira and A J Moreno (Totowa, NJ: Humana Press) 103-117

(8) Rottenberg H and Wu S (1998) Quantitative assay by fow cytometry of the mitochondrial membrane potential in intact cells Biochim. Biophys. Acta 1404:393-404

(9) Lu X, Kwong J Q, Molkentin J D and Bers D M (2016) Individual Cardiac Mitochondria Undergo Rare Transient Permeability Transition Pore OpeningsNovelty and Significance Circ. Res. 118: 834-841

(10) Wei-LaPierre L and Dirksen R T (2019) Isolating a reverse-mode ATP synthase-dependent mechanism of mitoflash activation J. Gen. Physiol. 151: 708-713

(11) Suraniti E, Vajrala V S, Goudeau B, Bottari S P, Rigoulet M, Devin A, Sojic N and Arbault S (2013) Monitoring Metabolic Responses of Single Mitochondria within Poly(dimethylsiloxane) Wells: Study of Their Endogenous Reduced Nicotinamide Adenine Dinucleotide Evolution Anal. Chem. 85: 51465152

(12) Vajrala V S, Suraniti E, Goudeau B, Sojic N and Arbault S (2015) Optical Microwell Arrays for LargeScale Studies of Single Mitochondria Metabolic Responses Mitochondrial Medicine Methods in Molecular Biology vol 1264, ed V Weissig and M Edeas (New York, NY: Springer New York) 47-58

(13) Vajrala V S, Suraniti E, Rigoulet M, Devin A, Sojic N and Arbault S (2016) PDMS microwells for multiparametric monitoring of single mitochondria on a large scale: a study of their individual membrane potential and endogenous NADH Integr Biol 8: 836-843

(14) Youle R J and van der Bliek A M (2012) Mitochondrial Fission, Fusion, and Stress Science 337: 10621065

(15) Tinevez J-Y, Perry N, Schindelin J, Hoopes G M, Reynolds G D, Laplantine E, Bednarek S Y, Shorte S L and Eliceiri K W (2017) TrackMate: An open and extensible platform for single-particle tracking Methods 115: 80-90

(16) Hollander J M, Thapa D and Shepherd D L (2014) Physiological and structural differences in spatially distinct subpopulations of cardiac mitochondria: influence of cardiac pathologies Am. J. Physiol.Heart Circ. Physiol. 307: H1-14 


\section{Figures}
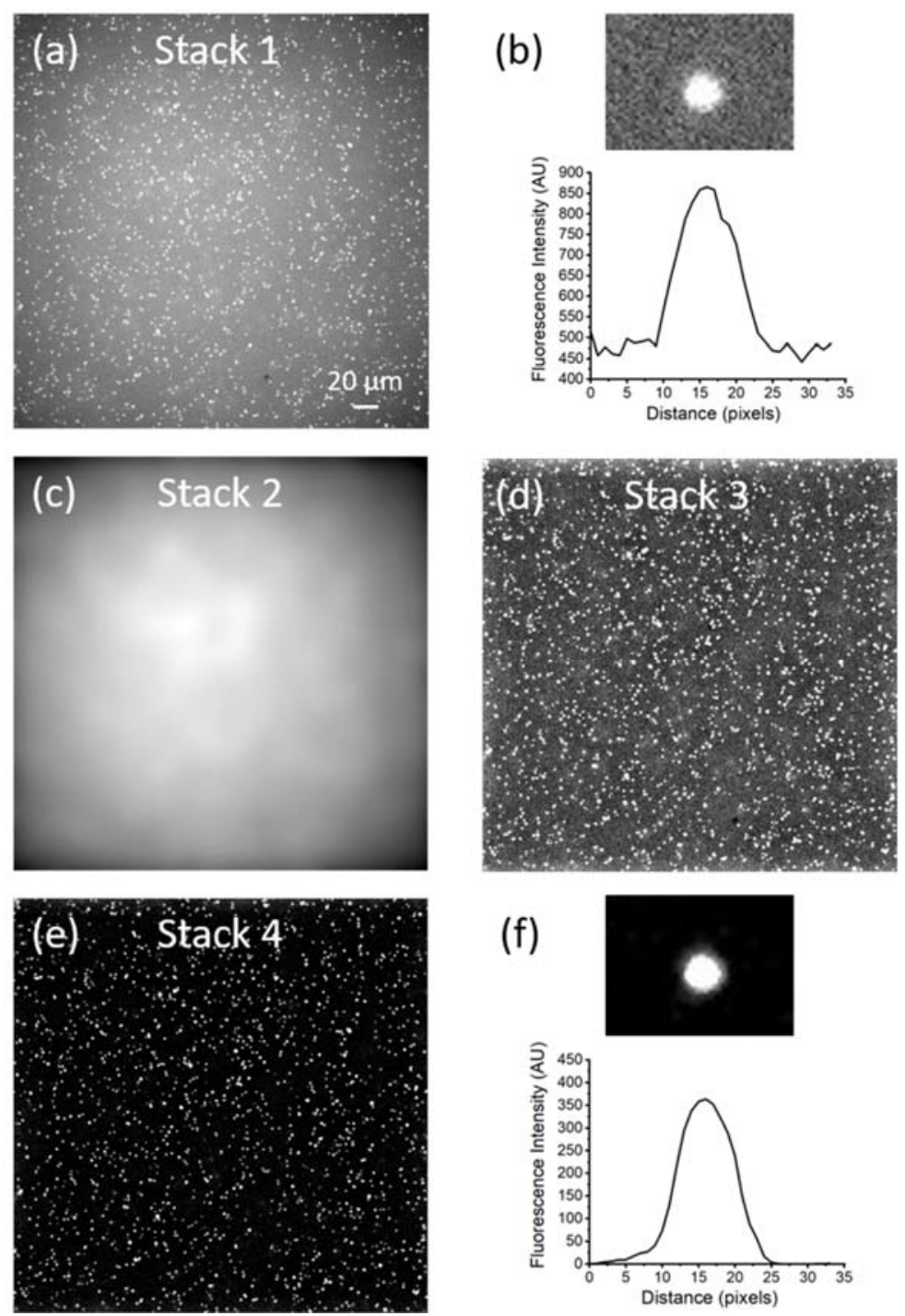

(f)
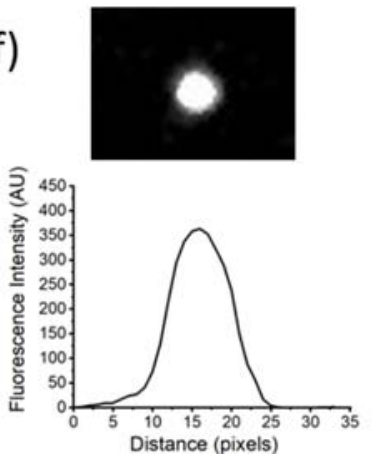

Figure 1: Protocol for the treatment of microscopy images with "Fiji" software. A) raw image of mitochondria after 20 min. sedimentation on a glass coverslip. Mitochondria were incubated with $10 \mathrm{nM}$ TMRM membrane potential dye and energized by the addition of Glutamate/Malate/Succinate at $5 \mathrm{mM}$. 10 images (every $10 \mathrm{sec} ., 40 \mathrm{~ms}$ exposure time) are used to create the Stack 1. B) example of a fluorescence intensity profile for a single mitochondrion of the image at this stage of the treatment. C) a Gaussian-type filter is applied (factor 60) to correct the shading effect due to the illumination inhomogeneity on the whole field, resulting images lead to Stack 2. D) Stack 2 is subtracted from Stack 1 to generate corrected images in Stack 3. E) Noise in the images of Stack 3 is corrected via a median filter 
(factor 2) and generates the final Stack 4. F) fluorescence intensity profile for a single mitochondrion, the same as in B), after the full image processing.

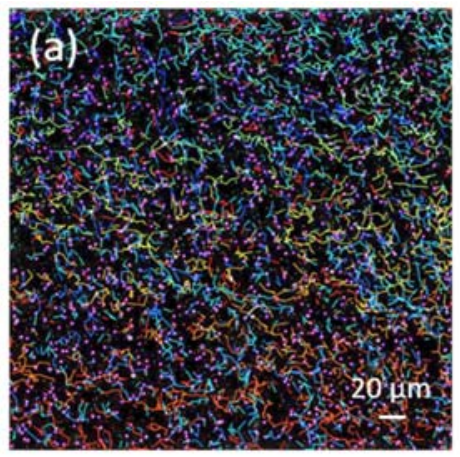

(b)

(c)
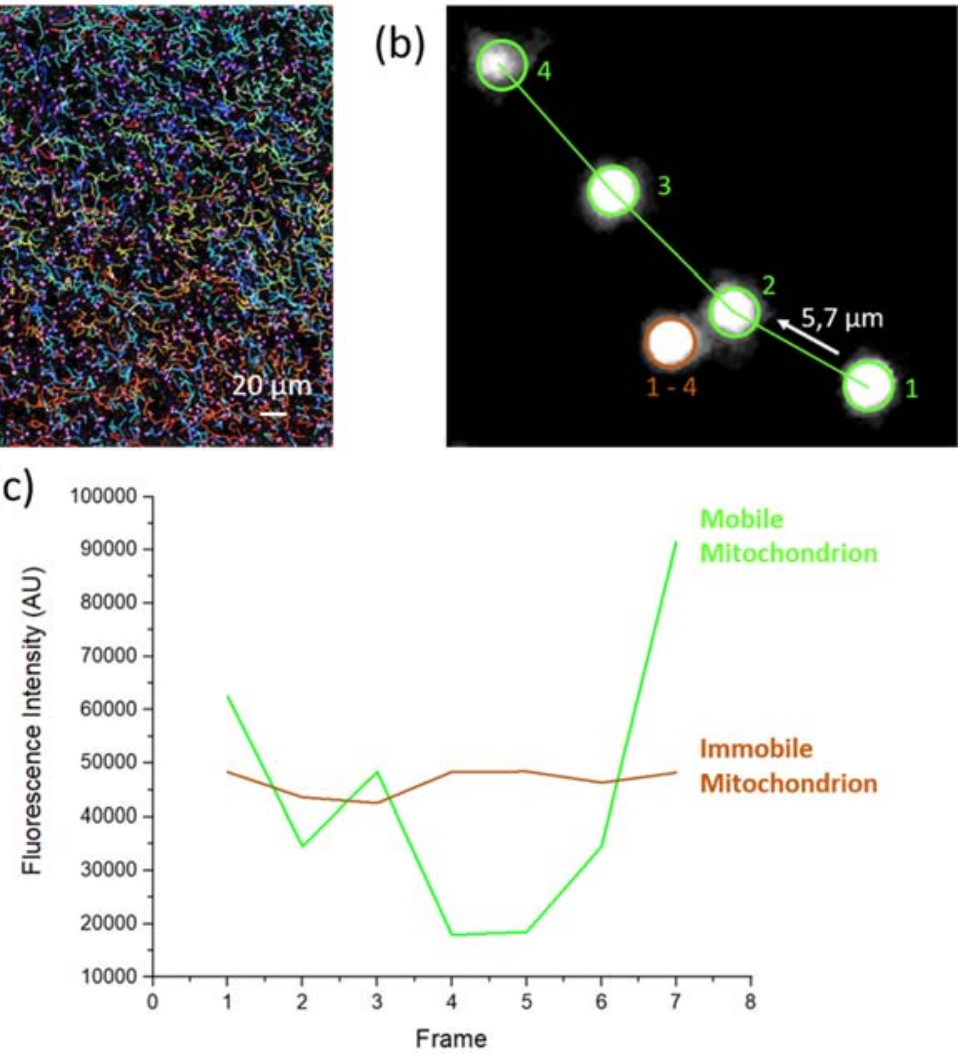

Figure 2 : Follow-up with the TrackMate plugin of "Fiji" software, of mitochondria mobility over time on the surface of a glass coverslip. A) Display of all trajectories (named tracks) from individual mitochondria during a sequence of 20 images (200 sec.); each object is identified and shown here with a colored trajectory. B) Shortcut of the whole image to display two mitochondria with very different behaviors along 4 images taken sequentially. C) Graph of the fluorescence variations due to displacements for the two mitochondria shown in B. Data treatments were applied on the images of the sequence in Figure 1. 

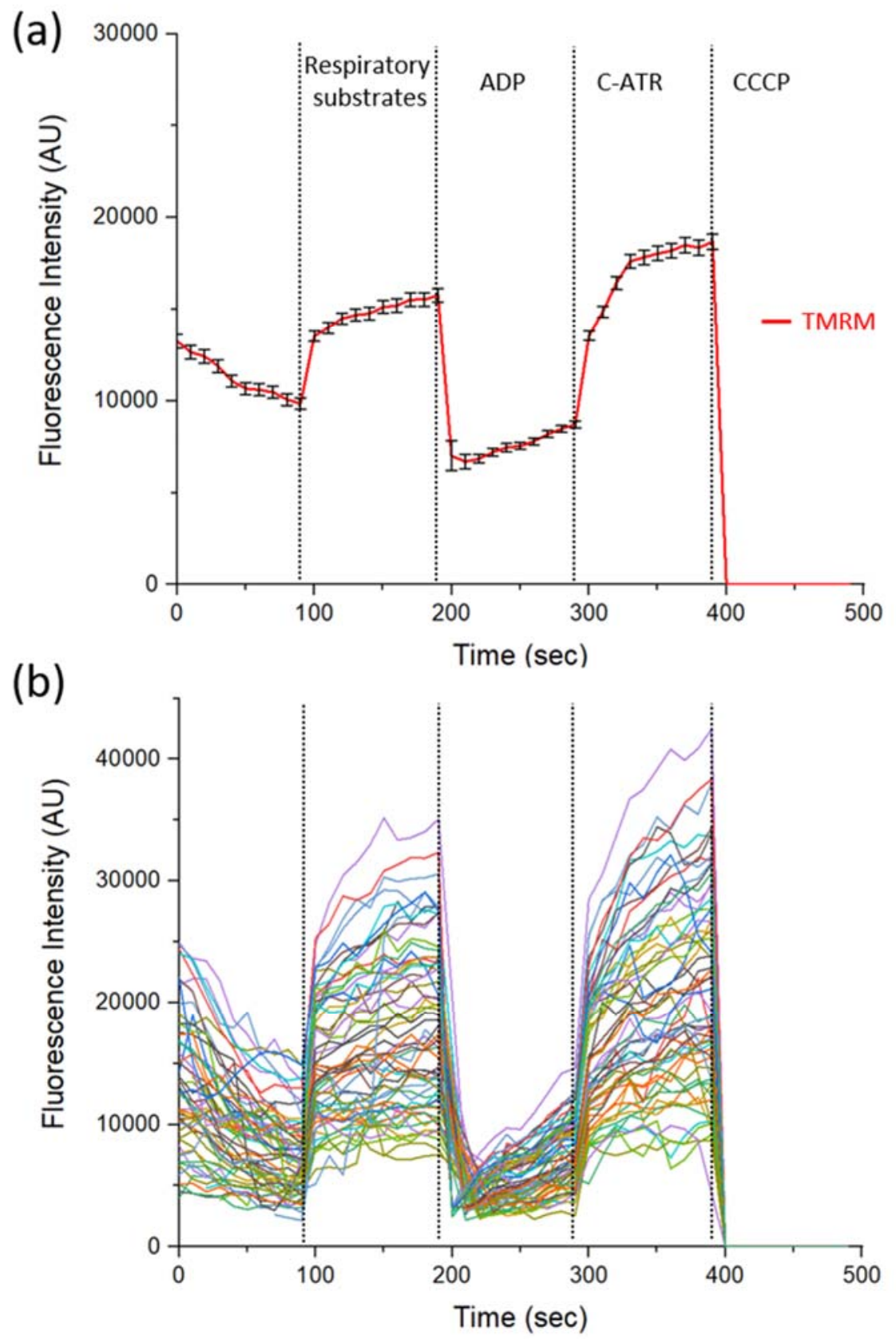

Figure 3 : Use of the image treatment protocol described in Figures 1 and 2 and of the specially developed scripts to monitor individual mitochondria responses within a population. A) calculation of the mean fluorescence intensity (mean \pm SEM) of all the mitochondria in each image to display the variations along a sequence of bioenergetics effectors. B) 100 individual mitochondrial responses overlapped which evidence the discrepancy of responses in a sample. The variations of the mitochondrial membrane potential were monitored by fluorescence microscopy with TMRM $(10 \mathrm{nM})$ for 20 minutes during the 
following sequence: $20 \mathrm{~min}$. sedimentation and immobilization on the glass surface; addition at $100 \mathrm{sec}$. of respiratory substrates (glutamate, malate, succinate at $5 \mathrm{mM}$ each); addition at $200 \mathrm{sec}$. of ADP at 1 $\mathrm{mM}$; addition at about $300 \mathrm{sec}$. of ATR at $5 \mu \mathrm{M}$; final addition at $400 \mathrm{sec}$. of CCCP at $0.5 \mu \mathrm{M}$. 